\title{
Transverse sternal plating in secondary sternal reconstruction
}

\author{
Joseph Huh, MD, ${ }^{\mathrm{a}, \mathrm{c}}$ Faisal Bakaeen, MD, ${ }^{\mathrm{a}, \mathrm{c}}$ Danny Chu, MD, ${ }^{\mathrm{a}, \mathrm{c}}$ and Matthew J. Wall, Jr, MD ${ }^{\mathrm{b}, \mathrm{c}}$
}

Objectives: Sternal instability after cardiac surgery occurs infrequently but can be challenging to manage. The most common treatment of mechanical sternal instability is operative rewiring. Transverse rib-to-rib stabilization with titanium plates designed for sternal fixation is a recent option. We evaluated the clinical utility of the transverse plating system in a cardiothoracic practice.

Methods: We retrospectively reviewed our experience with transverse sternal plating using the Synthes titanium sternal plate system (Synthes CMF, Paoli, Pa) from June 2004 to June 2007.

Results: There were 15 sternal reconstruction procedures performed in 14 patients using the transverse sternal plates. One patient required reoperation for sternal plate fracture. Indications for sternal plating were chronic sternal dehiscence in 9 procedures, acute sternal dehiscence in 3 procedures, previous mediastinitis and sternectomy in 2 procedures, and sternal plate fracture in 1 procedure. The initial cardiac operation was isolated coronary artery bypass in all patients. The mean age was 59 years (range, 51-68 years), all patients were men, 12 patients had a body mass index of greater than $30 \mathrm{~kg} / \mathrm{m}^{2}$ (range, $22-40 \mathrm{~kg} / \mathrm{m}^{2}$ ), 9 patients were diabetic, 8 patients were active smokers, and 7 patients had chronic obstructive pulmonary disease. The mean follow-up period was 15.5 months (range, 3-33 months). All patients achieved sternal stability with minimal postoperative pain. Complications included infection requiring explantation in 1 patient, plate fracture in 1 patient, and seroma formation in 1 patient.

Conclusion: The transverse sternal plating system successfully achieved stability in all cases and is valuable in complex sternal reconstructions. Sternal plating is an effective alternative to wire closure in sternum with fractures, chronic and acute instability, or poor bone quality.

Midline sternotomy is the most common access to the heart and mediastinum for cardiac surgeons. Stainless-steel wire closure remains the standard technique for stabilizing the sternum after sternotomy. Sternal complications have been classified as superficial if the drainage, dehiscence, or infection of the wound is at the skin and subcutaneous tissue. Deep sternal complications include dehiscence of the sternal bone, either because of mechanical forces or mediastinitis, and are fortunately uncommon. ${ }^{1,2}$ Rates of mediastinitis range from $0.4 \%$ to $6 \%$ in the literature. ${ }^{2-4}$ Sternal dehiscence and instability without infections are probably at least as common but more difficult to quantify because patients present over an extended period of time with chronic instability. Determining the extent of the instability or presence of infection can be difficult clinically because the appearance at the skin can be deceiving.

The most common treatment of mechanical sternal instability is operative re-exploration with rewiring. Wire techniques, such as the Robicek weave, can be used to reinforce osteopenic bone or overcome technical issues, such as off

From the Division of Cardiothoracic Surgery, Michael E. DeBakey Veterans Affairs Medical Center, ${ }^{a}$ Ben Taub General Hospital, ${ }^{\mathrm{b}}$ and Baylor College of Medicine, ${ }^{\mathrm{c}}$ Houston, Tex.

Joseph Huh reports lecture fees from Synthes Inc.

Received for publication Nov 15, 2007; revisions received March 5, 2008; accepted for publication March 22, 2008.

Address for reprints: Joseph Huh, MD, Department of Surgery, Michael E. DeBakey VA Medical Center, Baylor College of Medicine, 2002 Holcombe Blvd, Houston, TX 77030 (E-mail: jhuh@bcm.tmc.edu).

J Thorac Cardiovasc Surg 2008;136:1476-80

$0022-5223 / \$ 34.00$

Copyright $\odot 2008$ by The American Association for Thoracic Surgery doi:10.1016/j.jtcvs.2008.03.051 midline sternotomy. Unfortunately, the dehisced sternum that is rewired remains at high risk for subsequent breakdown. $^{5,6}$

Another approach for sternal stabilization includes rigid fixation systems. ${ }^{7,8}$ The stability of plate fixation systems has been demonstrated in orthopedic, plastic, and maxillofacial surgery but is not widely applied by cardiothoracic surgeons. Here we report our early experience with a sternal plating system that uses transverse rib-to-rib fixation (Synthes CMF, Paoli, Pa) in secondary reconstructions of the sternum. For plating purposes, we define secondary reconstruction as use of sternal plating in the setting of sternal complications, such as sternal dehiscence, sternal fracture, or previous sternectomy after initial primary closure. The Synthes titanium sternal plates have been designed with a safety release cotter pin at the midline to allow rapid reentry into the mediastinum in the event of early postoperative cardiac arrest after sternal closure. The transverse plates extend the area of fixation outside of the fractured sternum to the ribs laterally and overcome potentially problematic sternal bone quality. The titanium screws lock to the plates and provide added stability.

\section{MATERIALS AND METHODS}

We retrospectively reviewed our experience with the Synthes titanium transverse sternal plating system from June 2004 to June 2007 at the Michael E. DeBakey Veterans Affairs Medical Center. Institutional review board approval was obtained for this study. We used the plating system in secondary reconstructions of dehisced sternum after cardiac surgery or in difficult reconstructions after redo sternotomy. We included all patients who were treated with sternal plating for instability both in the acute setting during the same hospitalization after cardiac operations and in the chronic 


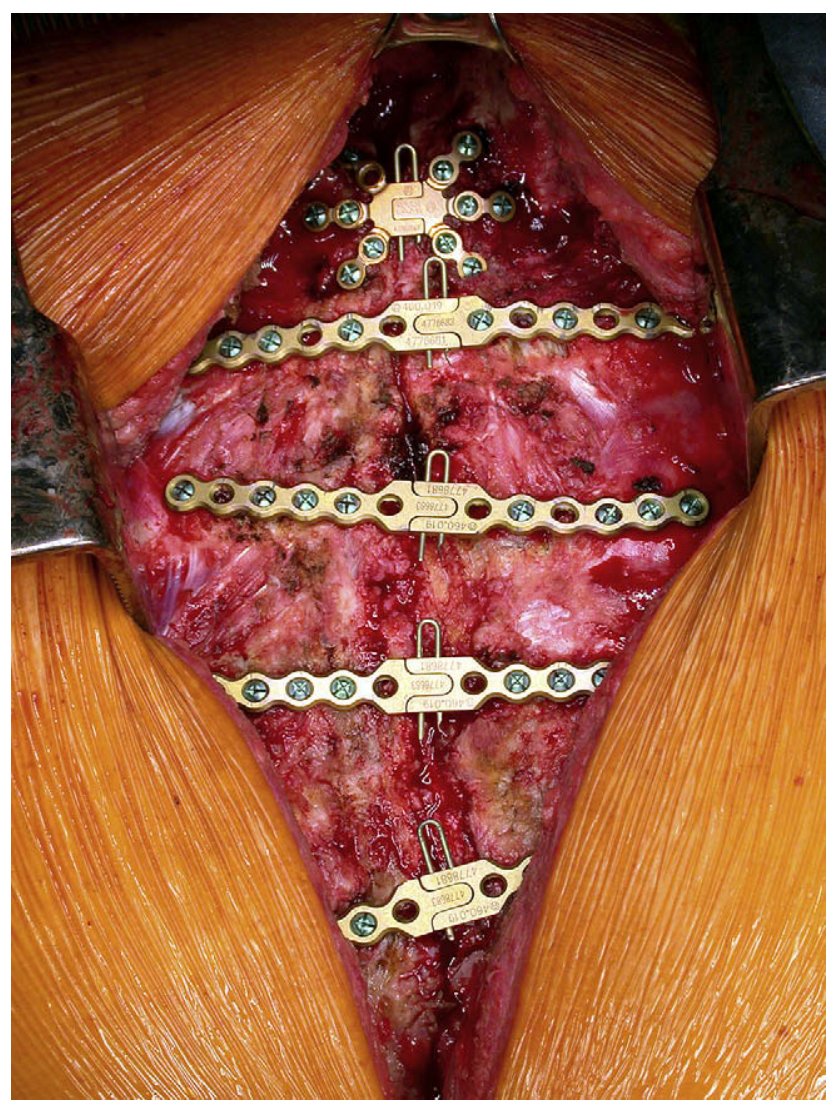

FIGURE 1. Sternal plates.

setting with long-term nonunion. The final decision to place the plating system was made intraoperatively at the time of sternal exploration. Frank purulence was a contraindication to sternal plating, and this occurred in 1 patient over the same time period who is not included in the current series. Patient demographics and follow-up data were obtained from the medical records.

The sternum was exposed through the previous midline incision. The degree of sternal bone damage was assessed. Swabs and tissue cultures were obtained from the bone, surrounding tissues, and any fluid that might have been present. Previously placed wires were cut and removed. The bone edges were debrided to achieve fresh bone-to-bone apposition when possible. Careful attention was made to prevent injury to the underlying cardiac structures. For repair of sternal nonunion, deep dissection below the sternum was not necessary. Pectoralis muscle flaps were raised, exposing the anterior chest wall. Reduction of the bone separation was achieved with reduction clamps. Transverse plates were then selected and appropriately contoured to the anterior chest wall. Usually, one manubrial plate was placed at the top of the sternum, followed by 3 to 5 transverse plates along the sternum (Figure 1). The screw holes were drilled with careful attention paid to the posterior table of the sternum and the lateral ribs to prevent injury to the underlying heart and lungs. The depth of the bone was measured with a depth gauge, and proper screw size was selected to achieve bicortical screw placement. Plates were fixed to the ribs and sternum with titanium screws and locked to the plates. The pectoralis muscle flaps were closed over round drains in the midline covering the plates. The overlying soft tissue and skin were closed primarily. Drains were kept in place until drainage was less than $30 \mathrm{~mL} / \mathrm{d}$. Patients were discharged with 3 months of sternal precautions. Postoperative chest radiographs were followed as indicated to assess for sternal plate integrity as well as other potential complications such as pneumothorax and hemothorax (Figure 2).

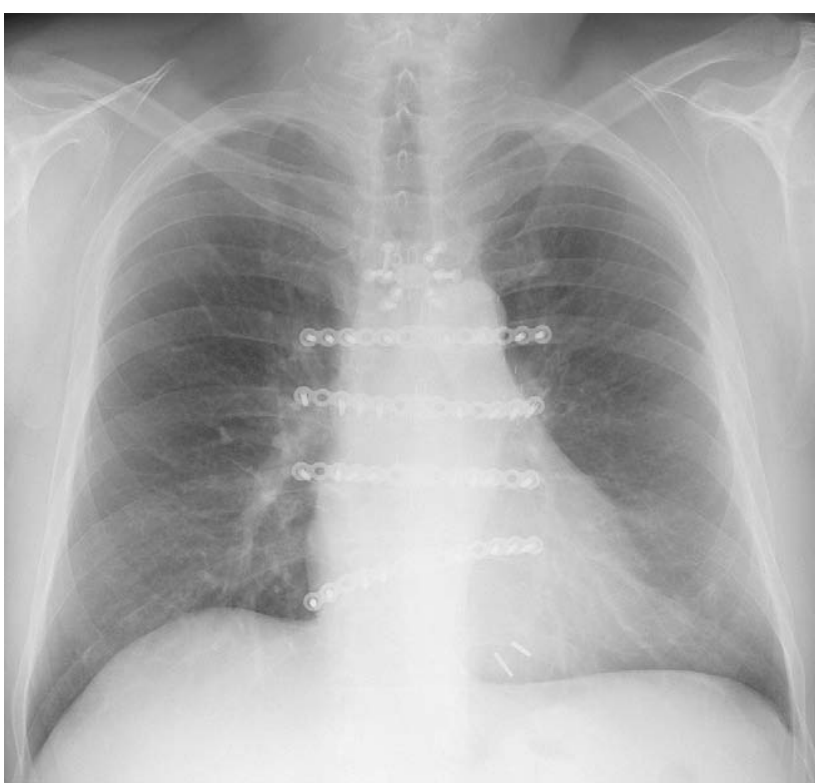

FIGURE 2. Postoperative chest radiograph.

\section{RESULTS}

There were 15 sternal plate operations performed in 14 patients to reconstruct the sternum. One patient required reoperation for sternal plate fracture. Three of the patients had their initial cardiac operations elsewhere and presented to our center with sternal instability. Indications for sternal plating with the Synthes transverse plating system are shown in Table 1. The initial cardiac operation was isolated coronary artery bypass in all patients. Preoperative patient risk factors and characteristics are shown in Table 2. The mean follow up period was 15.5 months (range, 3-33 months). All patients were alive at the time of follow-up.

The most common indication for sternal reconstruction was chronic dehiscence in 9 patients. Seven of these patients underwent initial cardiac operations at our center. The incidence of chronic sternal nonunion at our single center was $0.1 \%$ (7/715 cardiac operations over 3 years). The average time from initial cardiac surgery to sternal plating was 12.7 months for the 9 patients (range, 5-25 months). Of these patients, 4 reported a sternal click early at the time of discharge after their cardiac operations. The remaining 5 patients had instability at 2 to 6 months after the initial cardiac surgery. In 2 patients the instability was associated with falls and trauma to the sternum. All patients had gross instability at the time of plating, and the earliest stabilization was

TABLE 1. Operative indications

\begin{tabular}{lc}
\hline \multicolumn{1}{c}{ Indication } & 15 Operations \\
\hline Chronic dehiscence & 9 \\
Acute dehiscence & 3 \\
Previous mediastinitis & 2 \\
Fractured sternal plate & 1 \\
\hline
\end{tabular}


TABLE 2. Patient characteristics

\begin{tabular}{lc}
\hline \multicolumn{1}{c}{ Patient characteristics } & All patients $(\mathbf{n}=\mathbf{1 4})$ \\
\hline Mean age, y (range) & $59.6(51-68)$ \\
Mean weight, $\mathrm{kg}$ (range) & $106.6(175-305)$ \\
Mean BMI, kg/m $\mathrm{m}^{2}$ (range) & $34(22-40)$ \\
Mean albumin, g/dL (range) & $3.7(2.9-4.0)$ \\
Male sex & $14(100 \%)$ \\
Obesity (BMI $\left.>30 \mathrm{~kg} / \mathrm{m}^{2}\right)$ & $12(86 \%)$ \\
Diabetes & $9(64 \%)$ \\
Active smoking & $8(57 \%)$ \\
COPD & $7(54 \%)$ \\
Wheelchair dependent & $2(15 \%)$ \\
Prednisone use & $1(7 \%)$ \\
Previous mediastinitis & $2(15 \%)$ \\
\hline
\end{tabular}

$B M I$, Body mass index; $C O P D$, Chronic obstructive pulmonary disease.

performed at 5 months after cardiac surgery with failure to achieve union. One patient had a surgical site infection involving the hardware at 4 weeks after implantation, and the hardware was explanted. The sternum had gained enough union that he did not have boney instability with the removal of the hardware. The soft tissue wound was successfully managed with wound V.A.C. Therapy (KCI, San Antonio, Tex).

Three patients had postoperative sternal instability during the initial hospitalization after cardiac surgery. All 3 patients had wound drainage that was observed for 5, 14, and 30 days before re-exploration. The decision to proceed with the plating was made based on clinical assessment of the sternum for gross infection in the operating room. The presence of serous fluid, leukocytosis, and fever are all potentially confounding in the postoperative state and might not be reliable indicators of the presence or absence of deep sternal infection. Operative cultures of these 3 patients showed no growth.

In 2 patients plating was used to stabilize the chest after redo cardiac surgery in the setting of previous mediastinitis, sternectomy, and muscle flap reconstruction after initial coronary artery bypass surgery. One patient underwent redo coronary bypass, and the other underwent redo coronary bypass and aortic valve replacement. In both cases there was a gap in the sternum after the redo cardiac operation that was treated with sternal plating. The sternum was approximated at either the manubrium or at the lower sternal body to achieve bone-to-bone apposition at one point. The remainder of the sternum was plated across a gap. In both cases the patients achieved rigid sternal stability. The remnant of the previous rectus flap in both patients was placed into the bony gap. One patient had a protracted course after his second cardiac operation with lung failure requiring tracheostomy, as he did after his first cardiac surgery.

One patient underwent 2 separate plating procedures. He was wheelchair bound and obese $(129.3 \mathrm{~kg})$ and underwent his initial sternal reconstruction 2 years after his coronary bypass surgery. He had sternal instability within 1 month after coronary bypass at an outside institution, but because of his comorbidities, his sternum was not restabilized. He underwent sternal reconstruction with transverse sternal plating and achieved immediate stability, with improvement in his chronic pain. However, 5 months after his initial sternal plating procedure, he fractured his lowest plate and presented with acute onset of pain. Radiographs revealed the fracture at the interlocking tab at the safety cotter pin. At reoperation, the fractured plate was removed and replaced with 3 additional straight mandibular plates without the center interlocking tab. He has done well without subsequent complaints.

All but 1 patient were extubated either immediately postoperatively or the next morning after sternal stabilization. No pneumothorax or cardiac bleeding was encountered after sternal plating. Average length of stay after the sternal reconstruction was 15 days (range, 5-41 days). The wide variability was mostly due to waiting for drain outputs to decrease to $30 \mathrm{~mL} / \mathrm{d}$. There was one outlier because of ventilatory failure after redo coronary artery bypass.

No patient showed clinically significant restrictive pulmonary compromise with sternal and anterior chest wall fixation, although formal postoperative pulmonary function measurements were not obtained. All patients reported immediate stability and minimal pain. All patients with chronic nonunion had some degree of pain from the instability and reported prompt improvement in pain. Chronic postoperative pain was reported by 1 patient but was managed with oral nonsteroidal medications. We discussed removal of hardware with the patient at 2 years after plating, but he has opted to leave the hardware in place. One patient required drainage of a seroma at 2 months postoperatively. One patient had a surgical site infection involving the hardware and required explantation.

\section{DISCUSSION}

Sternal complications result in increased patient discomfort, disability, and mortality. Bony dehiscence and infection often occur together, and it is difficult to assess whether an infection led to the instability or vice versa. Inadequate bony fixation might lead to sternal osteomyelitis, and early postoperative instability might be a precursor to mediastinitis. Although sternal instability can arise from broken wires, the more common scenario is from the wires pulling through poor-quality bone, causing transverse fractures at multiple sites in the sternum. We believe that fixation of the sternal bone is indicated in reoperation to achieve union of the bone and prevent pain, regardless of whether this is achieved by rewiring, reinforced wiring (as with the parasternal Robicek weave), or plates. We suggest the use of plates as one alternative method to achieve boney fixation.

Rewiring the dehisced sternum is associated with a high rate of recurrent dehiscence, repeated operations, and 
continued pain for many patients. ${ }^{5}$ Rigid plate fixation has been advocated as an alternative in the reconstruction of sternal nonunion. ${ }^{9,10}$ Plate fixation has also been advocated for primary closure of sternotomy in selected high-risk patients. ${ }^{8}$ Cicilioni and colleagues ${ }^{11}$ recently used transverse plates that extended out laterally to the ribs, using mandibular plates for secondary reconstruction.

One advantage of the rib-to-rib transverse plating system is that it provides fixation beyond the sternum, which is often osteopenic and fractured in cases of sternal dehiscence. Additionally, plate stabilization is achieved on the anterior surface of the chest wall, and no dissection is necessary at the deep aspect of the sternum, avoiding risk to the underlying heart.

All patients in our study were men, representing the demographics of the Veterans Affairs patients. We do believe that the concept of rigid fixation beyond the sternum is applicable in women and the elderly, in whom the ribs might be osteopenic. The multiple plates spread forces over a wide area and allow a stable construct.

Our currently used plating system is designed specifically for the sternum and uses a safety cotter pin to allow emergent re-entry into the chest in the immediate postoperative period. The cotter pin release and all other quick-release methods of re-entry for sternal closure systems are only useful early in the postoperative course. Once bony union has occurred, releasing the cotter pin will not result in sternal opening. Cardiac surgeons planning for redo sternal entry in patients with healed plate closures should be aware that the plates would need to be removed to access the sternum.

Our case series represents reconstruction of mechanical instability without infection, although we could not confirm the absence of infection at the time of plating in the operating room until the cultures were finalized postoperatively. This is especially true in cases of acute postoperative sternal dehiscence, in which drainage and leukocytosis might confound the scenario. In each case the decision to place plates was made intraoperatively in the absence of gross purulence. During this study period, we had only 1 case of mediastinitis that was treated with conventional sternectomy and muscle flap reconstruction in consultation with the plastic surgery department. We would not advocate placement of hardware in an actively infected field. Several authors have successfully used staged sternal plate reconstruction after debridement of infection, open sternal wound care, and antibiotic therapy, followed by delayed plating once the gross infection had cleared. ${ }^{7,11}$ The pectoralis muscle flap is important in providing vascularized tissue coverage over the hardware to prevent infection.

Reoperations for plate complications were required in 2 patients who underwent operations for chronic dehiscences. A plate fracture and an infection necessitated revision or explantation of the plates within the first 5 months. In both of these cases, use of the plates did achieve boney fixation and a stable chest before removal. Whether traditional strategies, such as parasternal weave, in the setting of these cases of chronic sternal dehiscence would have achieved boney stability is uncertain. However, in a recent series of 48 patients with noninfectious dehiscence in which $86 \%$ of the patients were treated with Robicsek weave with or without muscle flap, there was a $14 \%$ need for reoperation and a $12 \%$ infection rate. Furthermore, $20 \%$ noted some ongoing instability, and $10 \%$ noted ongoing continuous pain. ${ }^{5}$ Several of our patients with chronic dehiscence were declined for sternal reconstructions elsewhere because of comorbidities and anticipated continued nonunion, and 1 patient had a failed prior Robicsek weave reoperation. In these patients we believed that the anterior transverse plates offered a simpler solution and more stable reconstruction. There has been no late failure or need for explantation of the plates to date in our series. Future studies that directly compare the various strategies of reconstruction in these difficult scenarios would be helpful to better define indications for sternal plating.

Comparative studies are also needed to address cost or cost-effectiveness of the plate systems. The average cost of implantation per procedure in our series was $\$ 8508$ (range, \$3900-\$16,189) and varied widely depending on the number of plates that were placed. Analysis of cost-effectiveness is difficult and cannot be deduced from this study. Comparison of cost with other methods of treatment, such as rewiring, failure of rewiring, and return to the operating room for failures, would have to be considered. Furthermore, improvement in stability and functional status or decrease in pain are subjective findings and might be difficult to quantify in cost analysis. Two of our patients underwent plating after redo cardiac surgery after previous sternectomy for mediastinitis, and a less expensive alternative to plating would have been to leave the anterior chest wall without stabilization.

Other potential complications include potential injury to the underlying lung and mediastinal structures. Care should be taken in penetrating the posterior table of the sternum and the ribs with the drill and measuring depth gauge. An accurately sized screw is selected from direct measurements. We had no incidence of pneumothorax or injury to the underlying heart for vascular structures.

Cardiothoracic surgeons using sternal plates should keep in mind that good reduction and bone-to-bone apposition is fundamental for long-term success. The hardware can break and fail with repeated motion of the chest, but if the bone heals under the plates, the chest will remain stable. Once sternal osteosynthesis occurs, the plate becomes extraneous and unnecessary for stability. We have placed plates across bony gaps in our series where there were large defects in the sternal bone, but every attempt was made to appose at least some segment of sternum to achieve bony stability. Bone grafts were used in small gaps, and adjacent ribs serve as suitable sites. 
We found the transverse plating system to be valuable to the cardiac surgeon. The cardiac surgeon most often carries out re-exploration of an unstable or draining sternum, and the plating system provides immediate intraoperative options. In addition, sternal plating systems can be used in primary or redo closures in which the quality of the sternum is poor or the comorbidities of the patient dictate alternative strategies over simple wires. Although techniques of molding plates, drilling bone, and placing screws might be unfamiliar to cardiac surgeons, the skills are easily acquirable in the context of the sternum.

These early to midterm results show transverse sternal plates to be an effective means of stabilizing the sternum. Complications noted occurred early in the course. Longterm follow-up is needed to determine the fate of the plates in the sternum. The small numbers in this retrospective report do not allow meaningful statistical comparisons, and this report serves as an observational series. The plating systems currently serve as an additional tool for the surgeon in the management of poor-quality sternum, in patients with multiple comorbidities, and for complications after sternal closure. Further experience and studies will be needed to better define the indications and limitations of plating systems in the sternum.

\section{References}

1. El Oakley RM, Wright JE. Postoperative mediastinitis: classification and management. Ann Thorac Surg. 1996;61:1030-6.

2. National Nosocomial Infections Surveillance (NNIS) System Report: data summary from January 1992 through June 2003, issued August 2003. NNIS System. Am J Infect Control. 2003;31:481-98.

3. Shroyer ALW, Coombs LP, Peterson ED, Eiken MC, DeLong ER, Chen A, et al. The Society of Thoracic Surgeons: 30-day operative mortality and morbidity risk models. Ann Thorac Surg. 2003;75:1856-65.

4. Immer FF, Durrer M, Mühlemann KS, Erni D, Gahl B, Carrel TP. Deep sternal wound infection after cardiac surgery: modality of treatment and outcome. Ann Thorac Surg. 2005;80:957-61.

5. Olbrecht VA, Barreiro CJ, Bonde PN, Williams JA, Baumgartner WA, Gott VL, et al. Clinical outcomes of noninfectious sternal dehiscence after median sternotomy. Ann Thorac Surg. 2006;82:902-9.

6. Peivandi AA, Kasper-Konig W, Quinkenstein E, Loos AH, Dahm M. Risk factors influencing the outcome after surgical treatment of complicated deep sternal wound complications. Cardiovasc Surg. 2003;11:207-12.

7. Plass A, Grünenfelder J, Reuthebuch O, Vachenauer R, Gauer JM, Zünd G, et al. New transverse plate fixation system for complicated sternal wound infection after median sternotomy. Ann Thorac Surg. 2007;83:1210-2.

8. Song DH, Lohman RF, Renucci JD, Jeevanandam V, Raman J. Primary sternal plating in high-risk patients prevents mediastinitis. Eur J Cardiothorac Surg. 2004;26:367-72.

9. Hendrickson SC, Koger KE, Morea CJ, Aponte RL, Smith PK, Levin LS. Sternal plating for the treatment of sternal nonunion. Ann Thorac Surg. 1996;62:512-8.

10. Bertin KC, Rice RS, Doty DB, Jones KW. Repair of transverse sternal nonunions using metal plates and autogenous bone graft. Ann Thorac Surg. 2002;73:1661-2.

11. Cicilioni OJ, Stieg FH, Papanicolaou G. Sternal wound reconstruction with transverse plate fixation. Plast Reconstr Surg. 2005;115:1297-303. 